\title{
Activités
}

$14-2$ | 2017

Dimensions et conditions d'une intervention capacitante

\section{Intervention et développement organisationnel en clinique de l'activité}

Le cas du dispositif « DQT » à l'usine Renault de Flins

Intervention and organizational development in clinic of activity. The case of the "DQT" device at the Renault Flins factory

Jean-Yves Bonnefond

\section{(2) OpenEdition}

Journals

Édition électronique

URL : http://journals.openedition.org/activites/3014

DOI : 10.4000/activites.3014

ISSN : 1765-2723

Éditeur

ARPACT - Association Recherches et Pratiques sur les ACTivités

Référence électronique

Jean-Yves Bonnefond, «Intervention et développement organisationnel en clinique de l'activité », Activités [En ligne], 14-2 | 2017, mis en ligne le 15 octobre 2017, consulté le 30 avril 2019. URL : http:// journals.openedition.org/activites/3014; DOI : 10.4000/activites.3014

Ce document a été généré automatiquement le 30 avril 2019.

\section{(c) $($ ) $(9)$}

Activités est mis à disposition selon les termes de la licence Creative Commons Attribution - Pas d'Utilisation Commerciale - Pas de Modification 4.0 International. 


\section{Intervention et développement organisationnel en clinique de l'activité}

Le cas du dispositif « DQT » à l'usine Renault de Flins

Intervention and organizational development in clinic of activity. The case of the "DQT" device at the Renault Flins factory

Jean-Yves Bonnefond

\section{NOTE DE L'ÉDITEUR}

Article soumis le 01/04/2017, accepté le 26/07/2017

\section{Introduction}

1 Cette contribution s'inscrit dans la perspective vygotskienne développementale que la clinique de l'activité mobilise. Pour Vygotski (2003), on le sait, il y a développement quand un fonctionnement devient le moyen d'un autre fonctionnement, il obéit alors à une migration fonctionnelle en changeant de statut (Clot, 2011 ; Kostulski \& Clot, 2007). C'est pourquoi on cherche ici du point de vue de la recherche à documenter le passage d'un fonctionnement organisationnel à un autre (Bonnefond, 2016) à partir du développement des objets, des interlocuteurs, des instruments dans l'activité des professionnels concernés (Clot, 2008a). Ce qui suit relève donc de ce qui est classiquement désigné comme une « recherche fondamentale de terrain » (Clot, 2008 b; Clot, 2017a).

En matière de santé et de performance, la question de la transformation des situations de travail et de l'organisation du travail qui y contribue est au cœur des enjeux économiques, sociaux et de recherches actuelles. Plusieurs disciplines, que l'on pourrait regrouper autour des « sciences de l'action », travaillent à trouver les voies pour étudier 
et améliorer l'efficacité et la santé au travail en faisant de celui-ci un objet d'élaboration par le dialogue.

3 C'est principalement le cas en ergonomie (Falzon, 2013), en psychologie du travail (Clot, 2010) et aussi en sciences de gestion (Detchessahar, 2013; Lorino, 2013). Les objets d'analyses et les modalités d'action varient, mais on y retrouve un problème partagé, celui des transformations organisationnelles pour l'amélioration du rapport entre santé et performance, à partir du travail concret.

Notre contribution à cette communauté de travaux en cours, tels qu'ils sont discutés aujourd'hui en psychologie du travail (Sarnin, Bobillier-Chaumon, Cuvillier, \& Grosjean, 2012), partage les préoccupations d'une ergonomie constructive (Falzon, 2013) pour des organisations capacitantes qui répondent aux exigences du travail contemporain, en visant le développement conjoint des individus, des collectifs et des organisations. Et ce, on le sait, alors même que du point de vue des prescriptions, nombre d'organisations sont théoriquement plastiques à l'expertise et l'initiative des salariés, sans y parvenir de manière effective (Perez Toralla, 2013). Ce résultat d'un travail réel dégradé dans des organisations théoriquement perméables à l'expérience professionnelle traduit une impossibilité organisationnelle à assumer la délibération sur les conflits de critères sur la qualité du travail (Clot, 2010 ; Clot \& Gollac, 2014).

Des travaux récents ont montré que les décalages entre performance prescrite par les indicateurs et celle "auto-prescrite» par les salariés ne pouvant être réduits complètement, c'est du ressort de l'ergonome que de contribuer à des organisations qui soient ouvertes à ces différences de point de vue pour agir sur les conditions de travail (Falzon, Nasciemento, Gaudar, Piney, Dujarier, \& Germe, p. 130).

6 Les conflits de buts (Caroly, 2002, 2010 ; Nascimento \& Falzon, 2009) nécessitant dans l'action des régulations de la part des professionnels, il s'agit de contribuer à la conception d'environnements de travail "non seulement adaptés et adaptables, mais " débattables", où les « inventions » quotidiennes des acteurs sont discutées et peuvent être intégrées à la structure de telle sorte que la conception se poursuive dans l'usage " (Arnoud \& Falzon, 2013, p. 224).

7 Dans cette perspective, Caroly \& Barcellini (2013) insistent sur les ressources du collectif de travail, et donc sur l'importance pour l'ergonome de travailler au développement d'une activité collective (Caroly, 2010), en équipant méthodologiquement le travail collectif de débat sur la qualité en situation réelle, à l'origine de réélaboration des règles. S'agissant spécifiquement des méthodes d'observation du travail (Bonnemain, Perro, \& Kostulski, 2015), des coopérations entre disciplines sont déjà ouvertes (Simonet, Caroly, \& Clot, 2011). Pour Daniellou, dans un dialogue interdisciplinaire récent, il ne s'agit plus que « l'expert débarque avec une meilleure organisation que la précédente. Il s'agit de donner des ressources aux acteurs pour qu'ils débattent et fassent évoluer les règles, il s'agit donc de soutenir le travail d'organisation » (Gaudart \& Rolo, 2015, p. 18 ; de Terssac, 2011). Sur cette voie, en tirant partie comme Coutarel, Caroly, Vézina et Daniellou (2015) des travaux de psychologie du travail et clinique de l'activité, Petit, Dugué et Daniellou (2011) invitent à une approche ergonomique qui donne une place active aux salariés dans l'intervention, au nom de l'efficacité, car ils sont les premiers experts, mais aussi au nom de leur santé.

On le voit, pour servir ces objectifs qui sont aussi les nôtres, la fonction de l'expertintervenant tournée vers la place active des salariés dans la transformation de 
l'organisation au nom de la qualité du travail et de la santé, est au cœur des enjeux d'efficacité d'intervention. Sur ce chemin, toute intervention rencontre nécessairement des problèmes méthodologiques de grande ampleur qui touchent aux rapports entre savoirs académiques et action de transformation, entre action et compréhension, entre dialogue et décision, entre pouvoir et pouvoir d'agir. Sans prétendre ici traiter ces vastes questions, on notera qu'il s'agit en tout cas, en accord avec les travaux ci-dessus, de dépasser les limites d'une action de «l'expert » qui vise à produire des connaissances sur le travail mises à disposition de directions " éclairées », créditées du pouvoir de prendre les « bonnes » décisions (Clot, 2015a, 2017a ; Kostulski, 2010). En effet, ce modèle - c'est un point crucial - ne permet pas d'expérimenter les transformations organisationnelles dans le cadre même de l'intervention.

Notre participation à ce dossier explore une voie possible pour répondre à ce problème. L'exemple mobilisé l'atteste à sa mesure, en matière de développement organisationnel, pour relier la santé - que nous référons au pouvoir d'agir - et la performance, il faut pouvoir expérimenter la transformation de l'organisation par les professionnels concernés au cours de l'intervention (Bonnefond, 2016). Pour documenter cette perspective qui procède d'une genèse instrumentale (Rabardel, 1995) en matière d'organisation, nous commencerons par présenter notre cadre théorique puis nous utiliserons l'analyse d'une expérimentation réalisée avec Renault à l'usine de Flins par l'équipe de psychologie du travail et de clinique de l'activité ${ }^{1}$ du centre de recherche sur le travail et le développement (CRDT) du CNAM.

À cette fin nous avons choisi de prendre une option chronologique et séquentielle qui permettra de suivre pas à pas le développement de l'intervention en question. Après avoir présenté son cadre et la méthodologie mobilisée, nous verrons donc comment des transformations ont été possibles.

11 Nous exposerons, depuis les analyses initiales sur les atteintes à l'efficacité et à la santé du travail d'opérateurs d'une unité de travail, comment «l'échafaudage » clinique peut donner lieu à des développements allant jusqu'à la conception et l'institution d'un dispositif organisationnel de dialogue sur la qualité du travail (DQT), instrumentant le dialogue et l'action sur les problèmes du travail. Ce dispositif, étendu à l'ensemble de l'usine aura trois ans d'existence à l'été 2017. Pour que de tels fonctionnements organisationnels puissent se pérenniser au-delà du retrait du cadre clinique, l'exemple mobilisé montre comment l'intervention instrumente différents paliers de développement de l'activité conjointe des opérateurs, avec la hiérarchie et la direction jusque dans la conception, la généralisation et le pilotage d'un processus organisationnel, et ce, dans ses dimensions technique, matérielle et sociale. Nous verrons que la conception et la généralisation de ce processus à l'usine s'est appuyé sur le développement organisationnel de la fonction psychologique et sociale du collectif (Quillerou-Grivot, 2011) au moyen d'une nouvelle fonction d'opérateur référent-métier désigné par élection pour six mois par ses collègues. Cette fonction organise ainsi un autre rapport social au réel qui permet d'enrichir celui inhérent à la division technique et hiérarchique du travail dans la production de compromis efficaces autour des conflits de critères sur la qualité du travail (Bonnefond, 2016; Clot, 2016a, 2017a). Ces transformations, que nous exposons plus bas, en équipant le dialogue et l'action sur les obstacles à l'efficacité contribuent ainsi à une performance réelle source de santé. Elles nous amènent à un double constat. D'une part elles permettent de confirmer la pertinence de la qualité du travail - interface de la santé et de la performance - comme 
une question institutionnelle centrale dans le travail contemporain, invitant à multiplier les expériences dans le domaine. D'autre part, ces résultats confirment l'importance d'une méthodologie qui fasse du développement personnel, collectif et aussi organisationnel à la fois l'objet et la méthode de l'intervention.

\section{Entre santé et performance : la qualité du travail et ses conflits de critères}

12 La clinique de l'activité emprunte à Canguilhem (2002) sa définition de la santé, qui n'est pas réductible à l'absence de maladie ou à une satisfaction affichée: «Je me porte bien, dans la mesure où je me sens capable de porter la responsabilité de mes actes, de porter des choses à l'existence et de créer entre les choses des rapports qui ne leur viendraient pas sans moi » (2002, p. 68). Santé et pouvoir d'agir sont donc parties liées chez ceux qui travaillent. C'est pourquoi c'est au chevet du travail que se porte une clinique de l'activité en se tournant vers l'action pour développer le pouvoir d'agir des professionnels, sur leur travail et sur eux-mêmes.

On comprend alors combien la santé est sensible à la qualité du travail, car elle est pour le sujet le moyen de parvenir (ou pas) à agir efficacement sur lui et sur le monde et ainsi de pouvoir se reconnaître dans ce qu'il fait (Clot, 2008a ; Tomàs \& Bonnefond, 2014). Dès lors, la santé au travail passe nécessairement par le soin apporté au travail lui-même (Fernandez, 2009) par l'implication des salariés, de l'encadrement et des instances de l'entreprise dans la résolution des problèmes d'efficacité de ceux qui y travaillent.

La qualité du travail, n'est donc pas un catalogue de solutions toutes faites à la disposition des acteurs, mais au contraire la recherche patiente et concertée de solutions adaptées au contexte particulier des entreprises.

De plus, parvenir à faire de la qualité du travail (Lhuilier, 2014) un objet de coopération entre direction, opérateurs, hiérarchie et syndicats dans l'organisation, est d'abord un problème avant d'être une solution (Quillerou-Grivot \& Clot, 2014) car le travail «bien fait » demeure l'objet de conflits de critères inévitables. La conflictualité est constitutive de l'activité même car le réel, dans ce qu'il a d'intrinsèquement inattendu, comme épreuve à laquelle on ne peut se dérober, ne cesse de « diviser » l'expérience, entre celle dont on dispose déjà et celle qu'on est en train de faire. Et ce, alors même que l'activité en cours est triadique (Clot, 2008a), pas seulement dirigée vers son objet, mais simultanément tournée vers l'activité des autres portant sur le même objet (collègues ou hiérarchie présente ou absente) et même vers les autres activités possibles du sujet. En cela, l'objet de l'activité d'un sujet appartient toujours aussi à d'autres destinataires et les buts qui s'y condensent ne se recoupent pas nécessairement. Il y a donc une sorte de collision intrinsèque à l'objet du travail où ces différents buts se mesurent, s'affrontent ou encore s'épaulent, rivalisent ou se dominent les uns les autres. Ce conflit propre à toute activité humaine vivante, jamais complètement éliminable, est la source inépuisable d'une régénération ou d'une dégénérescence, au moins potentielle, du rapport social aux objets du monde (Clot, 2008a ; Clot 2016a).

Or, on le sait, la vitalité de ce conflit - normal - dans l'activité à propos de l'objet du travail, qui débouche in fine sur des délibérations potentielles autour des critères du "travail bien fait», est en réalité largement malmenée dans les organisations contemporaines. Faute d'institutions dialogiques, ce conflit moteur se retourne souvent 
contre la santé et l'efficacité (Bonnefond, 2016; Clot, 2010, 2016b ; Clot \& Gollac, 2014 ; Tomàs \& Bonnefond, 2014). Le conflit entre critères, qui existe selon Leplat, lorsque «l'amélioration de l'estimation d'un critère conduit à la dégradation de l'estimation de l'autre» (2014, p. 22), ne se réduit pas à l'opposition spontanée entre exécutants et dirigeants. En effet, comme nous avons pu le montrer (Bonnefond, 2016) les conflits de critères sur la qualité du travail (par exemple, vitesse/précision, qualité/quantité) existent aussi bien dans l'activité personnelle, entre activités, dans les tâches prescrites et dans l'histoire du collectif.

Ce sont ces conflits de critères que l'intervention restaure, dans le cadre clinique par l'organisation du dialogue sur le travail. En renouvelant les objets et les instruments de discussions entre professionnels dans le collectif, puis entre eux et leur hiérarchie sur ces mêmes objets, on engage l'ensemble des protagonistes à éprouver les attendus habituels de leur expérience individuelle et collective pour que de nouveaux fonctionnements deviennent possibles. Dans de multiples traditions théoriques en psychologie, les conflits sont moteurs du développement (Dewey, 1975, 2014 ; Doise \& Mugny, 2004 ; Moscovici 1991, 1994 ; Vygotski 1994, 1997, 2003, 2014). Ils sont la source même du développement, ils l'expliquent car ils convoquent l'intelligence individuelle et collective. On ne peut s'y dérober même si leur destin n'est pas écrit d'avance.

La conflictualité autour des critères de qualité du travail fait donc de celle-ci un objet particulièrement riche, source - potentielle - de créativité donc de performance et de santé, à la condition de parvenir à coopérer sur cet objet dans l'organisation.

Or, notre exemple montre que, cette coopération dans l'asymétrie de la division technique et hiérarchique du travail, pour être effective, implique des moyens pour équiper le dialogue et l'initiative des salariés de première ligne en l'occurrence ici, les opérateurs d'usine.

À défaut, la paralysie du dialogue professionnel sur les « vérités » du travail réel gagne le métier, ce dernier s'ankylose et se fragilise dans l'activité de chacun et dans l'organisation tout entière. Les énergies sont alors dissipées et les ressources gaspillées. Une clinique de l'activité a pour objet de lever l'inhibition dialogique sur la qualité du travail par la reprise de la création professionnelle collective jusqu'à l'instituer dans de nouveaux fonctionnements organisationnels (Clot, 2010 ; Clot \& Gollac, 2014).

\section{Méthodologie d'intervention}

21 La méthodologie dialogique et développementale en clinique de l'activité est depuis son origine regardée comme moyen d'action afin de transformer le travail (Clot, 1999) et de produire en retour des connaissances sur ces transformations (Kostulski, 2010). Elle s'inscrit dans la perspective vygotskienne du développement où celui-ci n'est objet que s'il est aussi méthode car «c'est seulement en mouvement qu'un corps montre ce qu'il est » (Vygotski, 1978, p. 65). Il faut donc, d'abord "provoquer» le développement et transformer pour comprendre. On retrouve également ce primat de l'action dans la production de connaissance dans la perspective pragmatiste de Dewey, selon qui «il n'existe de vraie connaissance et de compréhension féconde que celles qui sont engendrées par l'action. » (1975, p. 327). Pour cet auteur, en effet « l'environnement doit être modifié pour être connu » (2014, p. 172). En conséquence, s'agissant des ressorts de l'organisation du point de vue du rapport santé-efficacité, on soutiendra qu'il faut 
s'efforcer de transformer cette organisation en la réindexant au travail réel, par le développement de l'activité des professionnels.

C'est pourquoi, en clinique de l'activité, la méthodologie que nous mobilisons intègre le registre impersonnel de l'organisation dans la dynamique développementale de l'intervention. Elle s'appuie sur un modèle du métier en psychologie du travail (Clot, 2008a) défini comme une institution, un rapport social composé de quatre registres qui ne peuvent s'agencer sans tension: personnel, interpersonnel, transpersonnel et impersonnel.

Les discordances créatrices ou destructrices entre ces registres et en chacun d'eux décident chaque fois de façon unique en situation, des propriétés concrètes du métier considéré. Le métier est impersonnel, sous l'angle de l'organisation officielle du travail, de ce qui est prescrit, de la tâche, des procédures, des outils, des fonctions, c'est le métier institué dans toutes ses dimensions formelles. Les registres personnel et interpersonnel concernent quant à eux directement l'activité instituante, vivante. Le métier est bien sûr personnel parce que toujours réalisé par l'activité pratique et psychique d'un sujet singulier, il est aussi irréductiblement interpersonnel car il ne se fait pas seul, il existe dans des activités conjointes avec d'autres. Ces deux registres concernent donc l'activité ordinaire toujours singulière, soumise aux aléas et aux exigences du réel. C'est à ce niveau que se réalise (ou pas) le dialogue au sein du collectif, sur la qualité du travail et donc que s'institue entre pairs le conflit de critères sur la qualité du travail. C'est ainsi que s'élaborent, se transmettent, s'évaluent et se renouvellent les différentes manières de faire et de dire pour rester efficace en situation réelle. Ce niveau est celui du collectif de travail, où vit (ou pas) la mémoire collective du métier, le registre transpersonnel et sa fonction psychologique d'instrument de l'activité. Ce registre a également une fonction sociale, car vivant dans les échanges sur les conflits de critères au-delà du périmètre du collectif de travail, il est également un instrument potentiel du maintien de l'organisation du travail au contact du réel et source de son développement possible.

L'intervention a alors ce double objectif : en premier, l'institution du conflit de critères sur la qualité du travail, comme moyen de développement du collectif de travail, par l'expérience faite par chacun des objections et controverses sur le travail bien fait. En second, l'institution du conflit de critères sur la qualité du travail au moyen du collectif de travail en dialogue avec la direction et la hiérarchie, pour développer l'organisation au service de la santé et de la performance. Trois phases y concourent.

La première débute à la demande d'intervention et consiste à construire l'instance dialogique, appelée ici comité de suivi, où pourra s'instruire le conflit de critères sur la qualité du travail en intégrant les instances officielles de l'entreprise (ligne hiérarchique, service de santé au travail, représentants du personnel). Y parvenir suppose des analyses et des données sur le travail réel et sa qualité, que seuls les professionnels réalisant le travail en question peuvent produire. C'est pourquoi ces derniers sont amenés à rejoindre cette instance dialogique après avoir analysé leur travail.

En effet, c'est l'objet d'une seconde phase que d'organiser entre professionnels volontaires la co-analyse de leur activité. Nous utilisons dans notre exemple la méthode des autoconfrontations croisées, largement documentée par ailleurs (Clot, 2005; Clot, 2008a ; Clot, Faita, Fernandez, \& Scheller, 2001; Kostulski \& Clot, 2007 ; Prot, 2006). Elle consiste à construire avec ceux qui le demandent, une analyse par eux avec nous de leur activité préalablement filmée. Cette méthode organise dialogues et controverses entre 
pairs sur la qualité du travail qui se traduisent par le développement de ressources collectives pour l'activité de chacun et la production d'analyses poussées sur le travail bien fait, ses problèmes, ses possibilités et les critères en jeu. La fonction psychologique du collectif de travail est ainsi revitalisée, au moyen du dispositif d'intervention, elle peut alors acquérir une fonction sociale de ressource pour l'organisation du travail. Le développement de cette double fonction du collectif, psychologique et sociale, ne vise pas uniquement à "restaurer » un dialogue dans le collectif ou dans l'organisation, il vise surtout à donner au dialogue un horizon de transformation des situations concrètes de travail problématiques par l'institution du conflit de critères sur la qualité du travail.

C'est l'objet d'une troisième phase où ces résultats produits par les professionnels sur la qualité de leur travail deviennent objet de dialogue avec eux dans l'instance prévue avec directions, représentants du personnel et professionnels de santé. À ce stade de l'intervention, dans ce cadre dialogique «extra-ordinaire», les possibilités de remise au travail de l'organisation se potentialisent sous l'impact de la qualité du travail réel analysée par les professionnels concernés. Alors, l'intervention peut s'ouvrir sur de nouveaux paliers de développement à franchir. L'ensemble de la méthodologie vise ainsi, en l'expérimentant, l'institution d'un professionnalisme, délibéré ${ }^{2}$ comme instrument durable d'une performance réelle donc source de santé (Clot, 2016 b, 2017 b ; Bonnefond, 2016). C'est dans cette perspective méthodologique qui met en son centre la place active des professionnels concernés que s'est réalisé le développement organisationnel que nous analysons maintenant.

\section{L'expérimentation à l'usine Renault de Flins}

\subsection{Un dispositif d'institution du dialogue sur la qualité du travail} des désaccords en impasse entre direction et organisations syndicales sur l'appréciation des situations de travail en matière de santé et de qualité. Après avoir refusé d'arbitrer ces désaccords, la proposition acceptée par tous, de l'équipe de psychologie du travail et de clinique de l'activité fût d'expérimenter, à plusieurs niveaux dans l'entreprise, des moyens de dialogue sur la conflictualité finalement « normale » des critères de qualité du travail (Clot, 2010), et ce, afin de construire avec les opérationnels concernés, des arbitrages organisationnels nouveaux qui soient sources de santé et de performance, en l'occurrence ici à l'usine de Flins.

30 Le dispositif initial de l'expérimentation articule trois principaux niveaux d'action. D'abord celui de l'atelier dans une UET (unité élémentaire de travail) du département montage choisie par la direction de l'usine, celle chargée de l'habillage des portes des voitures. Ensuite, celui de l'usine avec un comité local réunissant les organisations syndicales locales, mais aussi centrales, la direction usine et la ligne hiérarchique, la direction des ressources humaines usine et entreprise, le service de santé au travail, les intervenants du CRTD-CNAM et le moment venu, des opérateurs. Enfin, troisième niveau, 
celui de l'entreprise, avec un comité de suivi national qui réplique la même structure en ajoutant la direction de l'entreprise.

31 L'action du clinicien de l'activité commence par du temps passé au plus près du travail des opérateurs de la ligne des portes afin de construire avec ceux qui le demandent, une co-analyse de leur activité préalablement filmée au moyen de la méthode des autoconfrontations croisées; c'est le temps de la dispute professionnelle entre les professionnels concernés, sans participation de la hiérarchie. C'est le temps du travail collectif autour des plaisirs et des déplaisirs de la controverse sur le travail bien fait qui vise à développer la fonction psychologique du collectif pour imaginer de nouvelles possibilités de penser et d'agir. Ensuite, les résultats produits (sous forme de montage vidéo) font l'objet d'un autre dialogue au sein des comités de suivi. Ainsi, la fonction du collectif change de place dans le cours de l'intervention en devenant ressource pour modifier l'organisation du travail. En effet, la présence volontaire de ces opérateurs est consécutive à leur engagement dans le dialogue entre eux sur la qualité de leur travail au niveau de l'UET. C'est durant cette phase préalable que se construisent les conditions de leur contribution au dialogue sur le travail réel avec la hiérarchie et les syndicats en comité de suivi.

\subsection{La qualité du travail en analyse : dialogue refoulé et performance gâchée}

Précisons que l'UET d'habillage des portes compte environ 35 postes, 2 équipes se partageant les 16 heures de fabrication. Chaque équipe compte 35 opérateurs et 3 opérateurs séniors ${ }^{3}$ (OPS) avec à sa tête un chef d'unité (CU). Les opérateurs intérimaires représentent $80 \%$ de l'effectif dans une équipe, plus de $50 \%$ dans l'autre. Les portes arrivent nues dans l'UET, deux lignes de postes sont réparties le long de l'UET en face à face, avec d'un côté les postes des portes droites et de l'autre ceux des portes gauches. Ainsi, les portes sont successivement câblées, vitrées, habillées, équipées pour être ensuite acheminées dans une autre UET où elles sont remontées sur le véhicule en cours de montage sur la chaine principale. Il n'y a aucun temps de dialogue à proprement parler, la quasi-intégralité du temps de travail des opérateurs est directement productive, entrecoupée de deux pauses. Quasi intégralité du temps, car il est prévu des arrêts appelés " Animations ", arrêts hebdomadaires de 7 minutes et un mensuel de 20 minutes. Dans les faits, ces arrêts ne sont pas systématiques et sont facilement utilisés par la direction pour amortir des aléas de flux de production (par exemple lors d'une panne). Les chefs d'unité reçoivent alors sur leur messagerie un support informatique, parfois quelques minutes avant l'animation, indiquant les éléments à exposer aux opérateurs. Il s'agit de communication descendante, d'informations ou rappels de prescriptions diverses sur la qualité, la sécurité, les comportements, etc. Cela peut donner lieu à quelques questions-réponses, mais les temps et les sujets sont pratiquement toujours inconnus à l'avance et sont à l'initiative de la direction.

La présentation des enjeux de notre présence aux deux équipes a d'abord suscité de la défiance à l'égard d'une proposition d'intervention psychologique de la part de la direction de l'entreprise. Mais au bout du compte, après trois semaines passées au plus près du travail en ayant procédé à l'observation d'une quinzaine de postes, des opérateurs s'engageront avec curiosité dans ces travaux, alors même que la direction doutait de la possibilité de cet engagement dont d'ailleurs elle se félicitera. Ainsi, vingt d'entre eux, 
intérimaires et «Renault» répartis sur les deux équipes et regroupant dix postes de travail ont souhaité s'engager dans les autoconfrontations croisées. Ces dialogues et controverses sur la qualité du travail entre opérateurs, qui donnent lieu à un montage vidéo adressé au comité de suivi, ouvrent alors la voie au développement potentiel de la fonction sociale du collectif de travail et du dialogue dans l'organisation.

En effet, dans ces films, les dialogues entre opérateurs sur le travail - où les conflits de critères et les objections sont la source de la pensée - ont deux résultats simultanés dans le premier comité de suivi qui se déroule pourtant sans eux.

D'une part, la finesse des analyses produites au moyen des controverses professionnelles fraye un chemin à ces diagnostics très précis sur la qualité du travail réel adressés à la direction. D'autre part, le film «transporte» au sein du comité, la fonction de la confrontation des points de vue constitutifs du dialogue comme moyen de développement de la pensée, avec pour conséquence d'inviter les protagonistes du comité à faire entre eux ce qu'ils voient faire par d'autres au bénéfice d'une connaissance plus sérieuse du réel.

Ainsi, ont été donnés à voir et à entendre, d'une part l'engagement des opérateurs dans leur travail, la qualité de leurs analyses, leur ingéniosité, mais aussi les compensations parfois au prix de leur santé, des problèmes de conception de pièces, de moyens, d'organisation du poste et d'autre part, au plan du dialogue sur le travail, la «parole inutile »souvent éprouvée. À savoir, l'expérience répétée d'avoir parlé, signalé, proposé, sans que cela ne produise d'effets pratiques, au point que cette expérience de l'inutilité de la parole s'est transformée, au plan psychologique, en sentiment partagé (Poussin, 2014 ; 2015) d'impuissance organisationnelle.

37 Ce premier cycle depuis le travail collectif dans l'atelier entre opérateurs jusqu'à l'ouverture du dialogue, à l'échelon des comités de suivi entre direction, ligne hiérarchique et organisations syndicales, a posé les bases d'une institution du dialogue sur la conflictualité de la qualité du travail réel. L'engagement des opérateurs et la qualité de leurs analyses furent reconnus par les différents partenaires de l'intervention alors même qu'étaient simultanément attestés, l'inutilité de leur parole et le gâchis de performance.

38 On précisera ici que la fonction des intervenants est d'être garant du cadre dialogique qui devient une expérience organisationnelle vivante où les participants sont en situation d'être affectés par le réel du travail. Dans le cas présent, on le comprendra, la validité de ces diagnostics sur la qualité du travail, qui, en quelque sorte, "révèle» un sousdéveloppement de l'organisation, provoque chez ceux qui l'incarnent - hiérarchie et direction - un réel embarras. Ce problème de l'embarras et des affects dans le cadre clinique que nous mobilisons ne peut pas être sérieusement traité dans cet article. Il l'a été par ailleurs (Bonnefond, 2016; Bonnemain, 2015 ; Clot, 2015b ; 2016a ; Perrot, 2017). Précisons tout de même que ces inconforts ne sont pas écartés par les intervenants qui s'assurent alors de leur prise en charge dans le cadre dialogique car ils sont non seulement inévitables, mais nécessaires, ils sont de vraies « forces de travail » potentielles individuelles et collectives (Bonnefond \& Clot, 2016). En effet, les mouvements affectifs provoqués par l'intervention dans ces cadres sont la source d'énergie de ce que nous désignons dans le vocabulaire de Livet (2002), comme la "révision» potentielle des habitudes pour que de nouveaux fonctionnements soient possibles. 
e premier comité a ainsi permis à chacun d'être « au diapason » du travail réel, au moins du point de vue de ceux qui le réalisent et d'ouvrir sur une nouvelle étape destinée à agir sur ces obstacles à la santé et à la performance. Elle a consisté à expérimenter un dialogue entre encadrement et opérateurs sur la qualité du travail dans l'UET des portes, en partant de problèmes choisis par les opérateurs, comme nous allons le voir à partir d'un cas concret.

\subsection{Performance dialogique et pouvoir d'agir dans l'atelier : l'exemple des « coulisses »}

Dans l'atelier, alors que les postes ont été reconçus du fait du lancement d'un nouveau véhicule, nous avons organisé la mise en discussion de trois postes considérés comme particulièrement problématiques par les opérateurs avec l'encadrement de proximité (chefs d'unité et chef d'atelier). Les opérateurs de ces postes se sont engagés dans de nouvelles autoconfrontations croisées, mais cette fois les montages vidéos ont servi à dialoguer entre opérateurs et encadrement dans le but d'agir sur les problèmes soulevés. Ces nouveaux cadres dialogiques, conduits par les intervenants, ont produit des résultats, qui seront à la base des transformations organisationnelles qui s'ensuivront. Ils confirment que la performance concrète dépend de la performance dialogique (Bonnefond, 2016; Clot, Bouffartigue, Durand, Fortino, \& Mias, 2016), c'est-à-dire de dialogues au cours desquels se produit quelque chose de nouveau comme dans l'exemple qui suit (tours de parole 37-38).

41 Le cadre de l'expérimentation nous a servi à construire les conditions techniques et sociales de cette performance dialogique, pour que la conflictualité des points de vue sur la qualité du travail et les rapports de pouvoir hiérarchique soient dans une tension productive. C'est un point très important: dans le genre d'intervention que nous décrivons ici, les intervenants doivent être les garants d'un «primat du réel» sur le rapport social ${ }^{4}$, afin de faire obstacle à son refoulement éventuel, dans une forme fréquente de "retour aux habitudes». Ceci suppose, par exemple, que le psychologue intervienne dans les dialogues afin de nourrir le jeu des objections et anticiper sur le rééquilibrage des points de vue dans le rapport social spontané de subordination, comme l'attestent aussi Brunet, Pressein, Viel et See (2005). Les intervenants devant, en outre, entretenir avec l'encadrement et la direction, un dialogue sur ces questions à un autre niveau du dispositif.

Ainsi, grâce au jeu des objections entre opérateurs, qui leur a permis de diagnostiquer les obstacles à l'efficacité de leur propre travail, les opérateurs ont pu ensuite faire l'expérience avec leur encadrement d'un dialogue de qualité sur ces obstacles. Certains ont pu être levés par des transformations au poste, tandis que d'autres n'étaient plus refoulés, mais rendus objectifs dans l'échange, pour pouvoir être travaillés au-delà de ce périmètre. Pour l'encadrement ce fut également une ressource pour au moins deux raisons. Premièrement, la qualité des analyses et du dialogue a permis de répondre à des problèmes de performance initialement ignorés. Deuxièmement, il était possible à l'encadrement, de faire valoir ses arbitrages, expliquer les contraintes, les impossibilités qui sont les siennes, qui étaient tues jusqu'alors et parfois ignorées des opérateurs. Ainsi, des réaménagements de postes ont été traités, mais également des problèmes d'outillages ou d'engagement du poste ${ }^{5}$. Au-delà de l'UET, des transformations effectives ont 
également été réalisées, jusqu'à la reconception d'une pièce par l'ingénierie (Bonnefond \& Clot, 2016).

Prenons appui sur un des problèmes du poste des « coulisses ». Il s'agit de pièces de type joint qu'il faut monter à raison d'une par porte, dans laquelle la vitre coulisse. Elles font aussi office de joint entre la porte et la tôle de la caisse. La coulisse de porte-avant connait des problèmes de conception, conjugués à des problèmes de tôlerie. Plusieurs contraintes s'imposent ainsi dans l'activité de montage des opérateurs, qui pour y faire face, doivent notamment lubrifier la pièce afin de faciliter son insertion. La séance de dialogue opérateurs-encadrement sur ce poste s'est tenue avec les opérateurs des coulisses, dix autres opérateurs, dont deux opérateurs séniors (OPS) désireux de participer, les deux chefs d'unité (CU) et le chef d'atelier (CA). Cette séance particulièrement riche a contribué à plusieurs transformations.

Arrêtons-nous sur l'un de ces sujets, son développement dans le dialogue et les transformations qui s'ensuivront. Parmi les opérations à réaliser aux postes des coulisses, il faut apposer une mousse autocollante, mais la prise de cette mousse avec les gants mouillés, du fait de la lubrification, altère son caractère adhésif de sorte qu'elle ne tient pas et finit par tomber, engendrant alors des retouches à postériori. De plus, une autre pièce métallique appelée « écrou cage » est difficile à manipuler avec les gants mouillés, et déchire souvent ces derniers si bien que le lubrifiant entre au contact des mains, générant des problèmes de peau.

Dans la séance, on voit comment se construit la proposition de modifier l'engagement par la pose d'autres pièces (des «molaires »). Cela passe par l'explication du problème par des opérateurs, sans qu'ils aient de solution à proposer. Le chef d'atelier rejoint le diagnostic, reconnait ces difficultés et leur anormalité, il en explique les causes, ce que coûte la solution de la lubrification, et ce que l'encadrement fait avec la direction pour que cela puisse changer, mais ne voit pas comment faire davantage. Devant cette impasse, en difficulté, le chef d'atelier (CA) interpelle les opérateurs en sollicitant leur proposition (32), non sans injonction. L'opérateur réagit à cette injonction en confortant paradoxalement la position hiérarchique par un refus de s'impliquer davantage (33) dans la recherche de solution. Alors que la situation pourrait s'envenimer, nous allons le voir, ce dialogue empêché débouchera sur un échange avec quatre autres opérateurs, amenant à une proposition concrète et pertinente.

Cependant, cette proposition suppose la remise en cause de l'engagement de ce poste, impliquant du coup la remise en jeu de l'engagement d'un autre poste, ce qui fait naître une nouvelle confrontation dialogique. Le chef d'unité (CU) tente de faire valoir un statu quo (40) qui va engendrer une opposition directe d'un opérateur. Au final, le poste sera modifié.

Légende $:$ CU = chef d'unité - CA = chef d'atelier - OPS = opérateur sénior - OP1, OP2, OP... = opérateurs - Psy 1 = intervenant

1. OP1 : Tes gants, t'es obligé de les mouiller pour bien sortir la lèvre (de la coulisse). Après les mousses tu les prends comme ça, quand c'est mouillé la mousse elle tient pas. La plupart du temps, il y a des mousses qui tombent sur la chaîne, après les gens qui sont en bas ils remontent pour venir chercher des mousses pour en recoller. À chaque fois ça tombe, tu ne peux pas bien les mettre, quand t'as pas les gants secs tu ne peux pas. 
2. Psy1 : ça c'est vrai avec le savon, c'est pas vrai avec le produit?

3. OP1 : le produit aussi il n'est pas bon pour les gens qui travaillent à la chaîne. Moi j'ai eu des problèmes au début quand je suis rentré, il y avait des gants verts, mais ils étaient trop petits. Quand je les mettais au bout d'une demi-heure ça se déchirait, le produit rentrait. Quand je rentre chez moi en prenant la douche, je vois mes mains qui sont gonflées, j'avais un doigt j'ai eu un problème, vous voyez, c'était à cause du produit. Là maintenant il y a des gants c'est la bonne taille, mais ça gène toujours, faut trouver une solution.

4. OP2 (délégué syndical) : la solution c'est de revoir plutôt la coulisse et de la modifier de façon que tout ça soit supprimé. Qu'il n'y ait plus de liquide où quoi que ce soit. Il n'y aura plus ces risques-là, plus ces problèmes de collants qui ne collent plus. C'est la coulisse, qu'il faut modifier, qu'il faut revoir de façon que l'on puisse poser la coulisse sans avoir le problème d'enfoncer et qu'elle se monte bien.

5. CA : On est complètement d'accord, en fait le produit qu'on met sur la coulisse c'est une solution, provisoire, moi je la considère provisoire, ce n'est pas une solution définitive, on a des actions, tu (à psy1) as été à plusieurs réunions avec nous, où on a convoqué le central, vous avez même eu des visites, la personne qui était là cette semaine, c'est le directeur de la qualité Renault. Donc c'est pas n'importe qui, je lui ai montré qu'en fait la coulisse était in-montable en l'état et que l'on avait de l'eau savonneuse, que l'on avait du monopropylène glycol et que ça c'était une solution provisoire qui durait depuis quelques mois déjà et que l'on ne pouvait pas continuer dans cet état là. Déjà on n'arrive pas non plus à plaquer complètement la coulisse en partie avant, ce qui se voit dans les voitures finies, on voit que, quand on ferme la porte, en fait la coulisse elle plaque pas par rapport au montant.

\section{Plusieurs en même temps : oui, elle baille}

7. CA : Ça se voit dans la qualité client et dans la finition de la voiture. C'est vrai ce n'est pas un V1, mais c'est presque un $\mathrm{V}^{6}{ }^{6}$. Ca, un client il ne le voit pas, tous les clients ne le verrons pas, une partie des bons clients ils le verront. On a tout intérêt toute l'entreprise a un seul intérêt, c'est améliorer la qualité, mais la qualité va avec les conditions de travail. C'est pourquoi cette personne est venue, la direction de Flins l'avait convoquée, pour lui expliquer que l'on ne peut pas faire la qualité des véhicules si l'on a pas aussi des pièces qui se montent correctement. Donc complètement d'accord, mon problème c'est qu'aujourd'hui je n'ai pas de délai pour l'application de la solution définitive. C'est là où je suis en difficulté avec vous évidemment. Deuxième chose pourquoi le monopropylène glycol et pas de l'eau savonneuse. On a fait des mesures avec de l'eau savonneuse les efforts de montage sont de 13-14 kg. D'ailleurs si on ne met pas assez ou si ça sèche, vous montez à des efforts de 21-22 kilos on les a mesurés. C'est mesuré avec un dynamomètre. Le monopropylène glycol on descend à $9 \mathrm{~kg}$ d'effort. Ce qui n'est pas non plus...

8. OP3 : (opérateur aux coulisses) : Surtout sur la durée, il y a quand même 7h30 à forcer.

9. CA : On est d'accord 
10. OP3 : Avec de l'eau et avec le produit il y a une grande différence

11. CA : C'est pourquoi on a mis le mono-propylène glycol et je peux vous dire chaque bidon de 5 litres, on commande combien « OPS »?

12. OPS : 4 bidons par semaine

13. CA : 4 bidons par semaine, combien ça coute?

14. OPS : 80 euros

15. CA : 80 euros le bidon, fois 4 par semaine, vous voyez ce qu'on perd par semaine. C'est ce que j'ai montré au directeur de la qualité aussi. Je lui ai dit il y a ça, je lui ai montré aussi les lécheurs, on casse $25 \%$ des lécheurs c'est rebu, donc à peu près $1500 € /$ semaine. Donc, en fait il y a aussi un gain économique. Je vous dis, tout est lié, les conditions de travail ne sont pas uniquement des conditions de travail. Elles sont liées à la qualité et au coût.

16. OP2 : Oui et le mono propylène glycol le problème c'est que t'as des gars qui ont les mains abimées à cause de ça. Il y a un gars qui est venu me dire que quand il arrive chez lui, il s'enlève la peau.

17. Plusieurs : oui c'est vrai

18. CA : Qu'est-ce qu'on a fait ? On a commandé des gants verts qui vont dessous les gants normaux, donc on a un double gant, les gants verts qui protège la peau, les gants noirs qui protègent l'opérateur et en plus on a commandé une crème qu'on vous a donné,

19. Plusieurs : La crème on l'a jamais eu.

20. CA : Je l'ai amenée personnellement

21. Brouhaha

22. CA : Si il ya un problème de crème on en commande

23. Brouhaha

24. OP4 : Le problème c'est qu'on ne peut pas travailler avec les gants verts, ça nous gène. On ne sent pas les pièces, nous on a des petites vis à visser, tu le sens pas, tu fais ça ça tombe, tu perds le temps, on perd beaucoup de temps.

25. (plusieurs en même temps)

26. Psy1 : Pas tous, pas tous

27. OP4 : L'écrou cage !

28. OP2 : L'écrou cage ça va déchirer leurs gants.

29. OP4 : Et ça rentre dedans 
30. OP2 : Et le produit, ils ont le problème comme ils disent avec la mousse, quand tu touches, que t'as le malheur de toucher la mousse avec le produit et bien la mouse elle ne colle plus

31. OP4 : Ca glisse tu tiens l'écrou cage, ça tombe

32. CA : D'accord très bien, qu'est-ce que vous proposez ?

33. OP4 : Qu'est-ce qu'on propose, mais c'est vous !

34. OP5 : Ne pas mettre la mousse, mettre autre chose

35. OP4 : Oui, autre chose qui nous fait pas perdre du temps, parce que nous on perd beaucoup de temps

36. OP2 : Ceux qui utilisent le produit ne doivent pas utiliser les écrous cage

37. OP5 : On peut remplacer par les molaires

38. CA : Ca on peut regarder, bonne idée ! Ça c'est une bonne proposition

39. OP5 : Remplacer l'écrou cage par les molaires

40. CU : Après ça remet en cause tout l'engagement. Après, ... on peut, on peut regarder. Mais c'est pas sûr qu'on soit capable de le faire, c'est ça qu'il faut

41. OPS : Pareil pour les petites vis, faudrait les mettre ailleurs aussi

42. CU : Après ça re-chamboule tout,

43. OP6 : Il y a des problèmes de dextérité à cause de ça

\section{Brouhaha}

45. OP2 : Si il faut chambouler l'engagement on le chamboule hein c'est tout ! L'idée c'est de trouver des solutions!

46. Brouhaha

Le défaut de conception des coulisses perdurera malgré sa prise en compte par l'ingénierie, et l'engagement sera modifié. La mousse et « l'écrou-cage » seront remplacés par la pose de "molaires", des bacs de rétentions seront mis sous les supports des coulisses pour éviter le liquide sur le sol, l'implantation sera repensée en fonction de l'optimisation des déplacements de l'opérateur.

Au total, l'efficacité de ce processus dialogique, réalisé sur trois postes de l'UET, sera reconnue par les uns et les autres, opérateurs et hiérarchiques, tant du point de vue des problèmes traités que de l'amélioration des relations de travail et ce, malgré les tensions et confrontations liées à la conflictualité des critères de performance en jeu. C'est particulièrement le cas entre les critères managériaux de mesure de la performance économique théorique, et ceux d'une performance concrète au poste, amenant l'encadrement à devoir refouler le dialogue car les solutions vont dégrader les indicateurs de productivité théorique. C'est également le cas lorsque le traitement du problème est 
hors de portée de l'encadrement, on pense par exemple à la modification d'une pièce, ou au taux d'intérimaires qui fait obstacle à la polyvalence. Tout particulièrement dans ces cas, l'organisation du travail et les rapports sociaux habituels risquent de refouler le dialogue en engendrant une performance factice (Brunet et al., 2005; Bonnefond, 2016 ; Bonnefond \& Clot, 2016).

Ici, l'expérience était faite, que la confrontation des points de vue autour des critères de qualité est un moyen d'enrichir le travail lorsqu'elle peut disposer de ressources institutionnelles comme ici avec le dispositif clinique d'intervention. Alors les problèmes n'ont pas de raison de rester en l'état, ils peuvent se discuter et trouver des issues, comme ce fut le cas dans cet exemple. En outre, si le dialogue est un moyen, il est aussi un résultat car il permet de développer une expérience partagée "supportant» des problèmes du travail sans les évacuer, fussent-ils complexes à résoudre. Mais insistons, ces bénéfices du dialogue ne sont pas possibles sans résultats concrets sur le travail.

Ainsi, selon les propos de l'encadrement et ceux des opérateurs en comité de suivi, les relations professionnelles s'améliorent, l'ambiance de travail y gagne, il est plus facile de se comprendre, l'efficacité se développe. Enfin, et c'est un point déterminant pour la suite, des opérateurs, engagés dans le dispositif d'expérimentation au tronçon des portes sont devenus au fil du temps de plus en plus capables de discuter du travail avec leurs collègues, leur chef et au-delà, en comité de suivi. En un sens, l'exercice du dialogue sur leur activité avec leurs collègues et avec la hiérarchie en a fait des interlocuteurs de référence dans l'UET et au-delà.

\subsection{Du développement de l'activité au développement de l'organisation : des opérateurs référents élus}

51 L'évaluation de cette expérience dans l'UET « des portes » fut l'objet du second comité de suivi, nouvelle étape déterminante dans le développement du pouvoir d'agir des opérateurs, car elle leur offrit la possibilité de rentrer en discussion directement avec la Direction et les Organisations Syndicales à propos de cette étape franchie.

52 Le travail mené entre opérateurs sur leur poste, puis entre les opérateurs et l'encadrement de proximité, à l'instar de cet exemple des coulisses a conduit à plusieurs résultats concrets et à de meilleures relations de travail. La coopération nouvelle entre opérateurs et encadrement a ainsi pris la forme de conflits de critères institués particulièrement productifs. Mais les résultats ne sont pas que de cet ordre: ces opérateurs, engagés dans l'analyse de leur travail avec leurs collègues et dans le dialogue avec leur encadrement, ont acquis de nouvelles possibilités d'action sur eux et sur leur travail, devenant ainsi des interlocuteurs de référence sur la qualité du travail dans le cadre de l'expérimentation. Leur présence était donc nécessaire dans le comité local de suivi, en tant qu'interlocuteurs « experts » de leur travail auprès de la direction et des organisations syndicales. Ce comité réalise alors un développement de la fonction sociale du collectif de travail des opérateurs dans l'organisation, où celui-ci devient une ressource nouvelle dans l'activité de direction pour aller vers des transformations organisationnelles.

Les échanges sur ces résultats, considérés comme probants par tous, ont porté sur l'importance d'imaginer une organisation qui puisse généraliser l'expérience faite « aux portes ». Dès lors, plusieurs questions se sont posées. Outre les conditions matérielles de 
temps, d'espace et d'équipement du dialogue, comment faire vivre ces résultats sans la fonction «d'échafaudage » du dispositif d'expérimentation incarné par les intervenants du laboratoire de recherche CRTD-CNAM ? Comment équiper dans la durée l'initiative des opérateurs? Comment éviter «l'amnésie industrielle » des problèmes soulevés rendant de facto à nouveau la parole inutile ?

Des opérateurs expérimentés ont alors formulé la proposition de créer une fonction d'opérateur " référent ", qui serait élu par ses pairs pour être l'interlocuteur en matière de qualité du travail des opérateurs, à l'intérieur d'un processus à inventer. La direction a retenu le principe d'opérateur "référent", désigné par ses collègues et a décidé d'engager la construction d'un dispositif organisationnel, pour déployer à l'échelle du département montage, un processus de dialogue et d'action sur la qualité du travail qui s'affranchisse du périmètre expérimental de l'UET des portes. Ainsi, une fonction d'opérateur référent élu a-t-elle émergé comme une réponse au problème du passage de l'expérience réalisée avec les intervenants au tronçon des portes, à une organisation du travail généralisable qui équipe l'expertise et l'initiative conjointe des opérateurs et de la ligne hiérarchique.

55 À l'usine de Flins, nous avons vu que le pilotage existant de la performance, en considérant uniquement les critères " top-down ", amenait la ligne hiérarchique à éviter le dialogue et les arbitrages nécessaires à la performance selon les opérateurs (Bonnefond, 2016). Ce management basé sur les seuls pouvoir et savoir hiérarchiques même patiné d'une injonction à «l'écoute» - induisait alors de fait, une passivité hiérarchique devant les efforts nécessaires pour « manœuvrer » l'organisation du travail. Il a pu en être autrement « aux portes ", à partir du moment où, en plus du temps qui était accordé au dialogue, un poids était donné à la parole des opérateurs au moyen du dispositif de l'expérimentation incarné par les intervenants. Envisager de faire sans les intervenants ce qui s'est d'abord fait avec eux ${ }^{7}$, suppose donc de trouver les moyens institutionnels pour structurer durablement ce rapport dialogique au réel.

C'est à la condition de maintenir vivantes, les tensions et les contradictions entre critères de qualité dans les situations travail, qu'il est possible de faire des arbitrages et de prendre des décisions, fondées sur le développement et non sur le refoulement de la performance réelle.

57 C'est pourquoi, un autre rapport social au réel apparait comme une condition nécessaire pour imaginer une autre organisation technique, moins asymétrique, c'est ce que la fonction d'opérateur référent institue. Le réfèrent élu, ainsi légitimé par le collectif des opérateurs aussi bien que par l'entreprise qui organise l'élection, se trouve donc à prendre en charge, à incarner cette force de rappel du travail réel dans le dialogue avec ses pairs et avec la hiérarchie au moyen d'un processus organisationnel à construire pour que cette force de rappel fonctionne.

\subsection{Co-concevoir et tester un prototype organisationnel}

58 À cette fin, l'organisation technique à concevoir doit éviter d'éteindre cette motricité des conflits de critères, en équipant l'initiative des opérateurs afin que les développements de leur activité puissent s'inscrire dans l'organisation du travail.

59 En conséquence, la phase de conception de l'artefact organisationnel doit elle-même intégrer les opérateurs, afin de rester connectée au réel de leur activité d'une part et pour 
ne pas altérer la dynamique de son développement d'autre part. Aussi, parmi les opérateurs qui s'étaient engagés dans l'expérimentation jusqu'alors, deux d'entre eux de chaque équipe ont été désignés par leurs collègues pour participer avec l'encadrement à la conception de l'organisation modifiée, devenant des opérateurs référents de l'UET.

Pour mener à bien ce travail de conception et de test du processus en question, le directeur de l'usine a missionné, le chef d'atelier pour travailler avec l'équipe du CRTDCNAM, les quatre "référents» de l'UET des portes et les deux CU. Parmi ces quatre référents, l'un d'entre eux prendra particulièrement en charge la dynamique collective, sa fonction ne cessera de se développer. La fonction des intervenants-psychologues consiste quant à elle à soutenir et protéger ces développements contre l'inertie des fonctionnements existants. En effet, dans cette structuration inédite de la conception, l'action des intervenants demeure capitale pour garantir un cadre dialogique où prévaut le rapport au travail réel, dans l'asymétrie des rapports de hiérarchie. Ainsi, le chef d'atelier, les quatre référents, les deux chefs d'équipes et les intervenants du CRTD-CNAM vont constituer le groupe de conception d'un prototype organisationnel de la "performance dialogique " pour le traitement des "problèmes opérateurs", dont les principes seront les suivants :

- Au sein des UET, par équipe, une fonction d'opérateur référent élu par ses collègues opérateurs (le département compte alors plus de 600 opérateurs répartis dans 14 UET et 28 équipes).

- Une organisation temporelle et matérielle du dialogue sur les problèmes de qualité du travail entre opérateurs, entre référents et encadrement puis en réunion d'équipe.

- La formalisation, la hiérarchisation, l'orientation et l'enregistrement des problèmes.

- Leur traitement et leur suivi.

61 Ce travail de conception a été réalisé dans l'action, par alternance entre développement de l'activité et formalisation d'outils, de principes et règles pour structurer un cycle qui enchaîne un temps dédié à l'activité de référent pour le recueil des problèmes détectés et des solutions éventuelles proposées par les opérateurs, le déclenchement de leur mise en discussion, de leur traitement et les décisions contrôlées qui en découlent. On peut considérer qu'on assiste ici au développement d'un "environnement capacitant» (Falzon, 2008) fonctionnel dans le développement de l'activité, créateur d'artefacts. Au total, la structuration du prototype organisationnel - réalisée en conditions standard de production dans l'UET des portes - va durer six mois. Ce prototype prendra le nom de dispositif « dialogue sur la qualité du travail » (DQT) qui, à l'issue de sa phase de test, fera l'objet d'un comité local et d'un comité national validant son déploiement au-delà de l'UET, à l'échelle du département montage.

62 Au final, la structure de base (Figure 1) est la suivante: l'activité débute par le remplacement et la sortie de son poste de production de l'opérateur référent, élu par ses collègues. Rendu ainsi disponible, il passe de poste en poste afin de voir tous les opérateurs et afin de collecter ce qu'ils ont à dire de leur travail, les difficultés qu'ils rencontrent, les solutions éventuelles qu'ils imaginent.

Cette collecte mensuelle doit être complète, précise et formalisée par le référent. Dans un second temps, il rencontre son homologue de l'autre équipe, ils comparent et agrègent leurs données, en fonction des échanges avec leurs collègues et des sujets collectés (criticité, sécurité, ancienneté du problème, etc.) et préparent l'étape suivante, le rendezvous avec les deux chefs d'unité. 

des sujets discutés et collectés auprès des opérateurs, puis ceux qu'ils souhaitent voir traités en priorité. Cela donne lieu à des discussions sur l'analyse et sur les possibilités de traitement. Les problèmes sont ensuite catégorisés et orientés en fonction de leur complexité, vers le bon périmètre de responsabilité (chef d'unité, chef d'atelier, chef de département).

Les solutions possibles sont mises à l'agenda pour mise en œuvre, les sujets qui le nécessitent sont soumis à une analyse complémentaire, ou encore sont pris en compte et orientés vers l'instance de traitement adaptée. L'ensemble est enregistré dans une « liste unique de problèmes» (LUP), fichier informatique qui indexe chaque problème à une ligne, avec les différentes informations nécessaires (problème, catégorie, analyse, solution, délai, pilote, etc.). Ce fichier est ensuite affiché au sein des UET.

Figure 1 : schéma du processus « DQT ».

Figure 1: "DQT" process diagram
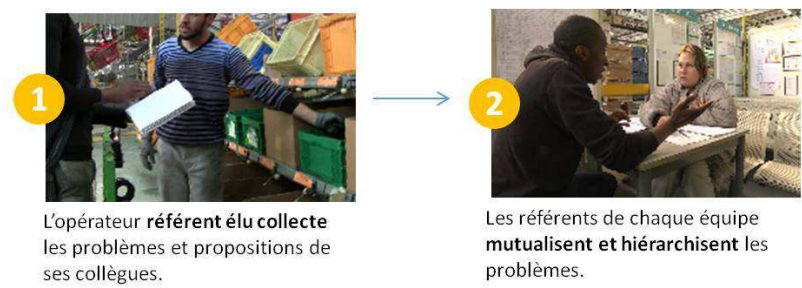

Les référents de chaque équipe ses collègues.

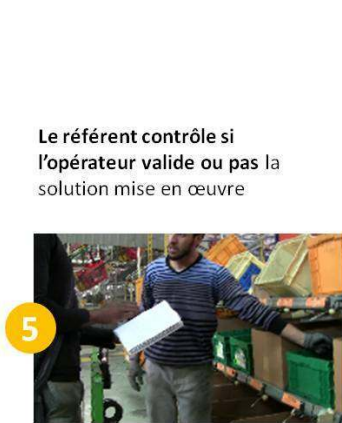
mutualisent et hiérarchisent les
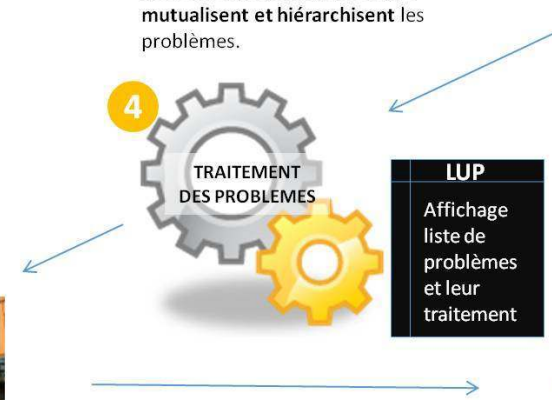

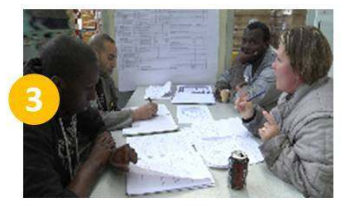

Encadrement et référents solutions aux problèmes, priorisation,
traitement. Enregistrement informatique

Réunion équipe entière Arrêt de chaine $20 \mathrm{mn}$ 2 fois/mois pour dialogue dans l'atelier sur les problèmes et solutions

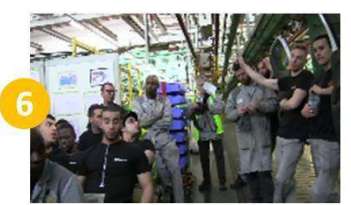
priorisation, orientation, pour

Le cycle se répète toutes les semaines. Jusqu'au mois suivant, la sortie hebdomadaire de chaine du référent est destinée à suivre le traitement des sujets, à voir avec les opérateurs concernés, s'ils valident les solutions mises en œuvre. C'est la condition pour clore un sujet avec l'encadrement lors du rendez-vous suivant.

Tous les 15 jours, des arrêts de chaîne de $20 \mathrm{mn}$ sont programmés pour animation «DQT ». C'est le moment où tous les opérateurs, le référent, et le CU discutent ensemble des sujets collectés, traités, en cours, et non traités. Cela se fait à l'aide de l'impression de la LUP affichée dans l'espace UET.

68 Ce prototype conçu et testé "aux portes", mais aussi discuté avec les syndicats et la direction a été validé en comité de suivi local, puis validé en comité national en vue de son déploiement dans l'ensemble du département montage d'abord.

\subsection{De la conception à la généralisation : le travail transformé}

69 Le déploiement sur les 14 UET du département montage se fera en commençant par la présentation du dispositif à la ligne managériale, assurée par le chef de département et le chef d'atelier en présence des intervenants du CRTD-CNAM. Afin de présenter le dispositif 
aux opérateurs, pour qu'ils puissent envisager les élections de référents et s'y préparer, deux arrêts de fabrication de 30 minutes furent programmés à deux semaines d'intervalle. Le premier arrêt étant destiné à la présentation du dispositif aux opérateurs par leur chef d'unité et le second pour procéder aux élections. Une fois celles-ci réalisées, la stratégie de déploiement de l'ensemble du processus restera développementale. Elle sera basée sur la transmission de leur expérience par les quatre opérateurs référents de l'UET des portes, à leurs collègues, nouveaux référents du département montage. Elle a été particulièrement efficace du point de vue de tous, à commencer par les référents formés et formateurs.

En effet, dans cet apprentissage, les référents expérimentés sont les seuls à pouvoir transmettre bien plus que la prescription, bien plus qu'un mode opératoire seulement impersonnel. La transmission n'a pas sa source dans le registre « impersonnel » délié de l'activité des opérateurs. Elle s'est faite par l'entremise de ceux-là même qui disposent à la fois de l'expérience d'opérateur commune à tous leurs collègues et du développement de cette expérience dans une nouvelle activité, une nouvelle organisation, ce qui leur confère beaucoup de moyens potentiels pour agir avec et sur leurs collègues.

Ces référents expérimentés se sont engagés dans cette nouvelle phase inédite, mais nourrie par la trajectoire développementale engagée antérieurement dans l'intervention avec les chercheurs sur leur activité et ses empêchements, jusqu'à l'expérimentation, la conception et la transmission de la fonction de référents dans l'organisation.

Cette trajectoire développementale sera la souche de la généralisation de cette nouvelle fonction. Le développement se réalise par alternance fonctionnelle, chaque activité réalisée devenant ensuite instrument d'une nouvelle activité au moyen des ressources collectives. Le développement a commencé pour les futurs référents, lorsqu'ils ont pris, avec d'autres opérateurs de l'UET des portes, leur activité comme objet de dialogue et de pensée entre eux, afin d'évaluer la qualité de leur travail et d'en faire ensuite le moyen d'affecter les activités de direction et syndicales en comité de suivi. Les résultats produits avaient ouvert sur une nouvelle activité dans l'UET, le dialogue avec l'encadrement à partir des analyses opérateurs sur les problèmes, faisant reculer la performance gâchée, provoquant ainsi la genèse de la fonction nouvelle de référent qui sera reconnue en comité de suivi. Ils devront ensuite mobiliser cette activité dans une autre activité qui prend pour objet l'organisation, afin d'y déposer en quelque sorte ces développements du pouvoir d'agir, transformés ainsi en marge de manœuvre organisationnelle (Clot \& Simonet, 2015). Ce faisant, cette organisation impersonnelle (cf. Figure 2 : principes DQT affichés dans les UET,) peut alors être en position d'instrument potentiel pour le développement de l'activité de tout opérateur. En sollicitant ainsi l'initiative tout en l'équipant, on considère comme capacitants ces développements organisationnels. 
Figure 2 : Principes «DQT » affichés dans les unités de travail.

Figure 2: "DQT" rules displayed in the work units

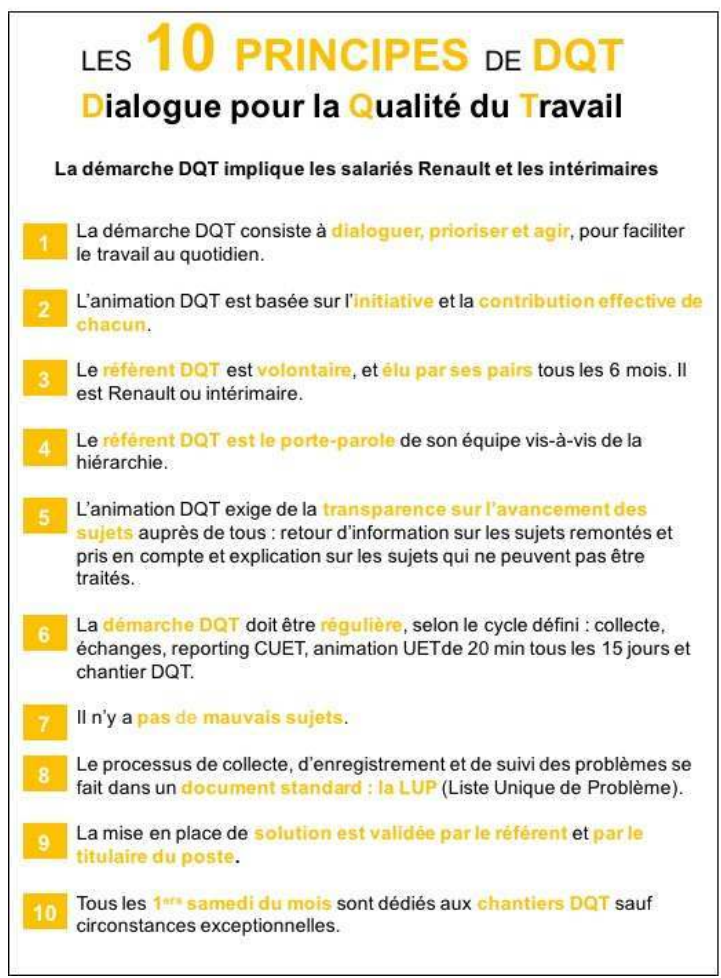

La phase de déploiement réalise ce développement organisationnel qui sollicite et équipe l'initiative. Les activités des référents « des portes », de conception et les précédentes, passent dans une nouvelle activité dont l'objet est cette fois-ci l'activité de transmission, de formation des nouveaux référents. Ce déploiement dans les 14 UET du département se réalisera en six semaines en conditions standard de production, l'ensemble du processus étant coordonné par le chef d'atelier avec l'appui des intervenants.

Dans cette phase de prise en charge par l'usine du transfert du dispositif dans les UET, la position clinique d'intervention doit également s'ajuster. Elle devient un accompagnement plus large qui mêle une présence constante et quotidienne, mais avec peu d'interventions directes dans les espaces où se déploie l'activité des référents, avec leurs collègues référents devenus formateurs et avec les chefs d'unité. L'intervenant est discret dans les interactions directes, mais très présentes au niveau du cadre de l'action. Il peut ainsi maintenir l'interférence du cadre clinique de l'intervention avec les fonctionnements de l'organisation existante en restant en position de ressources pour sa transformation.

\subsection{Le conflit de critères sur la qualité du travail institué dans l'usine}

Au plan de la conduite de l'intervention, le déploiement du dispositif « DQT » réalisé dans le département montage est un nouveau palier qui appelle désormais à un nouvel ajustement de la fonction du CRTD-CNAM. En effet, l'intervention a commencé par l'expérimentation dans l'atelier, puis l'usine a conçu et testé un dispositif avec notre appui. Désormais s'engageait le fonctionnement de ce processus en "vie série » pour 
reprendre le vocabulaire industriel, redéfinissant notre présence dans l'intervention et, de fait, notre fonction dans l'usine à travers une évolution de la conduite des comités de suivi. Ces derniers devenant une instance de suivi et d'évaluation de l'efficacité du dispositif tripartie: direction, organisations syndicales et, point crucial, référents. Le comité a inauguré cette fonctionnalité nouvelle avec neuf référents présents. Ainsi furent présentées les premières exploitations chiffrées des «LUP » qui indiquaient l'importance de l'investissement du dispositif et ces premiers résultats sur le travail. Mais au-delà des chiffres, ce fut l'évaluation par les référents eux-mêmes qui fit basculer le comité vers de l'inédit en cours de développement: par leur appréciation à la fois très positive du dispositif associé surtout par leur contribution directe dans les dialogues avec les participants du comité, en premier lieu avec la direction. À la suite des échanges qui ont eu lieu, les différentes organisations syndicales poseront, comme les référents, le problème de la "maintenance » et donc de la pérennité du dispositif. À cet égard, l'importance de la fonction de l'ancien chef d'atelier et de la fonction remplie par la référente formatrice de l'UET des portes sera soulignée, à commencer par les référents, conduisant à créer une fonction de "garant usine » et celle de "référente des référents " pour assurer cette maintenance "vie série». Les référents ne bénéficiant d'aucune "protection» particulière dans l'organisation, ces deux fonctions s'avèreront ensuite vitales pour le fonctionnement et le développement du dispositif dans l'usine. Le franchissement des étapes ultérieures doit beaucoup à l'installation de ces deux fonctions à vocation impersonnelle.

76 L'activité de "référente des référents» ne cessera de se développer par la suite, instituant ainsi les référents dans le système de veille et d'évaluation. Opératrice de l'UET des portes, elle sera peu à peu détachée pour d'une part, assurer la formation des référents, aider et accompagner les UET dans leur appropriation du dispositif et d'autre part, veiller au fonctionnement du dispositif, à sa maintenance, en coopération avec le garant usine de la Direction. Il est important d'insister sur l'articulation entre dialogue et décision. On sait, en effet, que par le passé, les initiatives en matière d'expression des salariés se sont heurtées à des obstacles organisationnels tenaces (Béroud, 2013 ; Bevort, 2013). Le processus de décision est une question centrale. Bernoux le rappelle: "Être empêché de participer aux décisions concernant son propre travail, c'est se voir refuser la reconnaissance de ce travail. C'est une injustice» (2015, p. 182). En ce sens précis, une clinique de l'activité cherche à faire reculer l'injustice pour améliorer la santé et les performances de l'activité qui ne sont nullement antagonistes par principe, même si elles sont conflictuelles par nature. À titre d'exemple, on peut mentionner les difficultés rencontrées dans la tenue des "rendez-vous ", aux différentes étapes du processus. On sait combien ces étapes actualisent, révèlent les conflits de critères dans chaque situation concrète où se jouent les arbitrages entre les contraintes du moment et les enjeux du dialogue et de l'action pour une performance durable. La référente des référents cherche alors à élucider avec l'encadrement les raisons des difficultés et à voir comment les dépasser, éventuellement en faisant intervenir le garant usine. Elle incarne à cet étage du dispositif la force de rappel des référents pour agir sur l'organisation afin de faire vivre le processus et maintenir un dialogue sur les difficultés aux différents niveaux de l'entreprise. À travers elle se joue donc une part de contrôle des référents sur le dispositif organisationnel et l'institution de cette fonction dans son pilotage avec la direction. C'est le cas également pour la gestion des LUP de l'ensemble de l'usine qui fait partie des responsabilités qu'endosse la référente des référents. Nous rappelons qu'elles sont 
imprimées puis affichées dans les UET, où elles sont annotées de façon manuscrite par les référents. Selon les dialogues réalisés, ces mises à jour font ensuite l'objet d'une saisie informatique pour être à nouveau imprimées et affichées.

Durant l'année 2015, un nouveau palier de développement aura lieu, le déploiement dans les autres départements de l'usine (Figure 3). Sans participation directe du CRTD-CNAM, il sera entièrement pris en charge par l'usine, réalisé par la « réfèrente des référents » qui sera détachée à plein temps à cette fin, toujours sous la direction du "garant du dispositif ». En décembre 2015, l'usine comptera 81 référents répartis dans 43 UET.

Figure 3 : Tableau de bord usine des résultats du dispositif « DQT » ${ }^{8}$ janvier 2016.

Figure 3: Factory Scoreboard of "DQT" Results - January 2016

\begin{tabular}{|c|c|c|c|c|c|c|c|}
\hline Entité & Montage & $\begin{array}{c}\text { CSC- } \\
\text { Qualité }\end{array}$ & Peinture & Emboutissage & Tôlerie & Logistique & \\
\hline Depuis & 19 Mois & 11 Mois & 10 Mois & 10 Mois & 2 Mois & 2 Mois & \\
\hline UET(s) & 12 & 1 & 8 & 6 & 10 & 6 & \\
\hline Référents & 23 & 2 & 16 & 14 & 18 & 8 & $\mathbf{8 1}$ \\
\hline $\begin{array}{c}\text { Sujets } \\
\text { ouverts }\end{array}$ & 1714 & 48 & 217 & 296 & 146 & 62 & $\mathbf{2} 483$ \\
\hline $\begin{array}{c}\text { Sujets } \\
\text { Traités }\end{array}$ & 1251 & 16 & 96 & 75 & 23 & 0 & $\mathbf{1 4 6 1}$ \\
\hline $\begin{array}{c}\text { Sujets } \\
\text { Sensibles }\end{array}$ & 15 & 1 & 0 & 14 & 1 & 0 & $\mathbf{3 1}$ \\
\hline
\end{tabular}

À partir d'une demande syndicale retenue par la Direction de l'usine, une « commission tripartite DQT » de suivi du dispositif fut créée en avril 2015, afin que l'usine développe son propre instrument de dialogue social sur la qualité du travail, avec les seuls représentants syndicaux de l'usine, sans la Direction de l'entreprise et sans l'équipe du CRTD - CNAM. Cette initiative s'inscrit alors dans la perspective du «démontage» de l'échafaudage de l'intervention et des comités de suivi. Ce transfert montre un développement de l'activité syndicale et de direction, mais aussi des référents, il institue dans la durée la conflictualité des critères de qualité du travail comme moyen d'un dialogue social renouvelé par l'activité des référents. Cette commission tripartite DQT réunit la direction de l'usine, la fonction $\mathrm{RH}$, le garant usine du dispositif, les organisations syndicales usine et la référente des référents, (à terme un référent de référents par département).

Enfin, ajoutons qu'en mars 2017 le dispositif s'est étendu à d'autres périmètres de l'usine et compte 107 référents. Mais surtout il a intégré des mécanismes d'incitation salariale. En effet, le nouvel accord triennal (2017-2020) sur l'intéressement des salariés de l'usine de Flins est composé d'un indicateur "DQT », indexé sur le nombre de chantiers dédiés aux traitements des problèmes collectés. Ce dispositif au service de la santé et de la performance continue donc à se développer en produisant de nouveaux fonctionnements organisationnels, indépendamment du cadre d'intervention initial CRTD-CNAM. 


\section{Conclusion}

80 Ainsi, les transformations organisationnelles que représentent ensemble ces fonctions, instances et processus, contribuent à attester de la motricité du conflit de critères sur la qualité du travail pour relier performance et santé au moyen du dialogue. Le dispositif «d'entrée » et «de sortie » des problèmes que la LUP incarne est à ce titre un bon indicateur du développement car sa dynamique dépend du dialogue, elle en est la trace dans le temps et pour chaque unité de travail. Simultanément, ces transformations montrent la nécessité d'un équipement institutionnel pour supporter dans la durée cette motricité.

81 Les instruments organisationnels produits conjointement, opérationnalisent l'institution du travail de qualité, ils constituent ainsi selon nous, un environnement capacitant, au sens « des environnements non seulement adaptés et adaptables, mais "débattables", où les "inventions" quotidiennes des acteurs sont discutées et peuvent être intégrées à la structure de telle sorte que la conception se poursuive dans l'usage » (Arnoud \& Falzon, 2013, p. 225). Dans la perspective vygotskienne mobilisée ici, il y a développement quand un fonctionnement devient un moyen d'un autre fonctionnement, il obéit alors à une migration fonctionnelle en changeant de statut (Vygostki, 2003). D'action en tant que telle, il devient opération au service d'une autre action et subit alors une sorte « d'automatisation organisationnelle ». En considérant que l'histoire du développement depuis le début de l'expérimentation, a pour objet le conflit de critères sur la qualité du travail, on peut lire ces fonctionnements organisationnels nouveaux comme un développement de ce conflit, une levée progressive et partielle de son déni, réversible, mais contenue, enrichissant cet «objet lien» dialogique (Tosquelles, 2009) qu'est l'activité source de performance et de santé.

Enfin, en matière d'intervention, les résultats obtenus dans cette expérience permettent d'étayer l'importance d'une méthodologie qui fasse du développement, à la fois l'objet et la méthode de l'action. De plus, ils documentent une voie quant au problème de la généralisation et de la pérennisation de transformations basées sur l'initiative professionnelle comme facteur de santé et de performance. En effet, comme nous l'avons $\mathrm{vu}$, répondre à ce problème suppose le développement d'un autre rapport social au réel du travail qui tienne compte de l'asymétrie de la relation hiérarchique inhérente à la relation salariale. Insistons sur ce point: nous avons montré que cette transformation organisationnelle tire sa pérennité d'une force de rappel nouvelle, l'opérateur référentmétier élu par ses pairs et en dialogue aussi bien avec sa hiérarchie qu'avec les instances représentatives du personnel. Il incarne une légitimité nouvelle susceptible de faire « descendre » l'organisation et les acteurs de l'ainsi nommé «dialogue social», sur les problèmes ordinaires du travail réel.

$83 \mathrm{Au}$ regard de la performance et de la santé, la genèse de cette fonction élective fait reculer, selon nous, ce que le juriste $\mathrm{C}$. Wolmark a désigné comme la « cécité du droit sur l'activité de travail » (2016, p. 168), cet « angle mort » de la relation salariale sur la qualité du travail, que notre intervention a modestement contribué à réduire.

84 À la lumière de cette expérimentation, mais aussi d'autres en cours ${ }^{9}$, on peut penser que le « professionnalisme délibéré » nécessaire au développement durable de la performance et de la santé dans l'organisation, implique donc de "prendre des libertés » avec le droit 
existant pour développer le droit du travail (Clot, 2016 b ; 2017 b ; Wolmark, 2016). C'est ce qu'ont décidé ensemble tous les partenaires de cette expérimentation.

\section{BIBLIOGRAPHIE}

Arnoud, J., \& Falzon, P. (2013). La co-analyse constructive des pratiques. In P. Falzon, (Ed.), Ergonomie Constructive (pp. 223-236). Paris : PUF.

Bernoux, P. (2015). Mieux-être au travail : appropriation et reconnaissance. Toulouse : Octarès Béroud, S. (2013). Perspectives critiques sur la participation dans le monde du travail : éléments de repérage et de discussion. Participations, 1(5), 5-32.

Bevort, A. (2013). De Taylor au néo-management : quelle participation des salariés ? Participations, 5(1), 33-51.

Bonnemain, A. (2015). Les paradoxes de l'intensité affective dans l'autoconfrontation. L'exemple de l'activité dialogique des chefs d'équipe de la propreté de Paris. Thèse pour le doctorat en psychologie. Paris : CNAM

Bonnemain, A., Perrot, E., \& Kostulski, K. (2015). Le processus d'observation, son développement et ses effets dans la méthode des autoconfrontations croisées en clinique de l'activité. Activités, 12 (2). En ligne. URL : https://activites.revues.org/1111

Bonnefond, J.Y. (2016). L'intervention dans l'organisation en clinique de l'activité. Le dispositif «DQT» à l'usine Renault de Flins. Thèse pour le Doctorat en psychologie. Paris : CNAM.

Bonnefond, J.Y., \& Clot, Y. (2016). Les affects et leur destin dans l'intervention. Activités 13(2). En ligne. URL : https://activites.revues.org/2895

Brunet, R., Presselin, J., Viel, M., \& See, N. (2005). Le risque et la parole - Construire ensemble une prévention des risques du travail dans l'agriculture et l'industrie. Toulouse : Octarès.

Canguilhem, G. (2002), Écrits sur la médecine. Paris : Seuil.

Caroly, S. (2002). Différences de gestion collective des situations critiques dans les activités de service selon deux types d'organisation du travail. Perspective interdisciplinaire sur le travail et la santé 4(1). En ligne.

Caroly, S. (2010). Activité collective et réélaboration des règles: des enjeux pour la santé au travail. Document d'habilitation à diriger des recherches en ergonomie, Université de Bordeaux 2, France.

Caroly, S. \& Barcelini, F. (2013). Le développement de l'activité collective. In P. Falzon, (Ed.), Ergonomie Constructive (pp. 33-45). Paris : PUF.

Clot, Y. (1999). La fonction psychologique du travail. Paris : PUF.

Clot, Y. (2005). L'autoconfrontation croisée en analyse du travail : l'apport de la théorie bakhtinienne du dialogue. In L. Filliettaz, \& J.P. Bronckart (Eds.), L'analyse des actions et des discours en situation de travail (pp. 37-55). Louvain-la-Neuve : Peeters.

Clot, Y. (2008a). Travail et pouvoir d'agir. Paris : PUF. 
Clot, Y (2008 b). La recherche fondamentale de terrain : une troisième voie. Éducation permanente, 177(4), 67-77.

Clot, Y. (2010). Le travail à cœur. Pour en finir avec les risques psychosociaux. Paris : La Découverte.

Clot, Y. (2011). Théorie en clinique de l'activité. In B. Maggi, (Ed.), Interpréter l'agir : un défi théorique (pp. 17-39). Paris : PUF.

Clot, Y. (2015a). De Mayo à Oddone. L'instruction au sosie. Préface de I. Oddone, A. Re \& G. Briante. Redécouvrir l'expérience du travail (2è éd.). (pp. 7-34). Paris : Éditions sociales.

Clot, Y. (2015 b). Les affects et l'action, préface à La fonction psychologique du travail (6è éd). Paris : PUF.

Clot, Y. (2016a). Activité, affect : sources et ressources du rapport social. In M. A. Dujarier, C. Gaudart, A. Gillet \& P. Lenel. (Eds.), L'activité en théories. Regards croisés sur le travail (pp. 51-80).Toulouse : Octarès.

Clot, Y. (2016 b). Clinique, travail et politique. Travailler, 36(2), 91-106.

Clot, Y. (2017a). Conclusion - L'intervention : entre terrain et laboratoire. In A. L. Ulmann, A. Weill-Fassina \& T. H. Benchekroun (Eds.), Intervenir - Histoires, recherches, pratiques. (pp. 185-190).Toulouse : Octarès.

Clot, Y. (2017 b). Travail et affectivité : un chantier ouvert, préface à Travail et pouvoir d'agir (2è éd). Paris : PUF.

Clot, Y., Bouffartigue, P., Durand, J.P., Fortino, \& Mias, A. (2016). Questions autour de la clinique de l'activité, La nouvelle revue du travail, 9. En ligne.

Clot, Y., Faïta, D., Fernandez, G., \& Scheller, L. (2001). Les entretiens en autoconfrontation croisée : une méthode en clinique de l'activité. Éducation permanente, 146, 17-27.

Clot, Y., \& Gollac, M. (2014). Le travail peut-il devenir supportable? Paris : A. Colin.

Clot, Y., \& Simonet, P. (2015). Pouvoirs d'agir et marges de manœuvre. Le Travail Humain,78(1), 37-49.

Coutarel, F., Caroly, S., Vézina, N., \& Daniellou, F. (2015). Marge de manœuvre situationnelle et pouvoir d'agir : des concepts à l'intervention ergonomique. Le Travail Humain,78(1). 9-29.

Detchessahar, M. (2013). Faire face aux risques psycho-sociaux : quelques éléments d'un management par la discussion. Négociations, 19(1), 57-80.

Dewey, J. (1975). Démocratie et éducation. Paris : Armand Colin.

Dewey, J. (2014). Reconstruction en philosophie. Paris : Folio.

Doise, W., \& Mugny, G. (2004). Psychologie sociale \& développement cognitif ( $2^{\mathrm{e}}$ éd.). Paris : Armand Colin.

Falzon, P. (2008). Enabling safety : Issues in design and continuous design. Cognition Technology and Work, 10(1), 7-14.

Falzon, P. (2013). Pour une ergonomie constructive. In P. Falzon, (Ed.), Ergonomie Constructive. (pp. 1-15). Paris : PUF.

Falzon, P., Nasciemento, A., Gaudart, C., Piney, C., Dujarier, M.A., \& Germe, J.F. (2012).

Performance and quality of work : an empirical assessment. Work, 41, Supplement 1. 3855-3860.

Fernandez, G. (2009). Soigner le travail. Itinéraires d'un médecin du travail. Toulouse : Erès. 
Gaudart, C., \& Rolo, D. (2015). L'ergonomie, la psychodynamique et les ergodisciplines. Entretien avec François Daniellou. Travailler 34(2), 11-29.

Kostulski, K. (2010). Quelles connaissances pour l'action en clinique du travail ? In Y. Clot \& D. Lhuilier (Eds.), Agir en clinique du travail (pp. 13-27). Toulouse : Erès.

Kostulski, K., \& Clot, Y. (2007). Interaction et migration fonctionnelle : un développement en autoconfrontation croisée. In Y. Clot \& K. Kostulski, Dialogue, activité, développement, Psychologie de l'interaction, 23-24, 73-109

Leplat, J. (2014). Aperçu sur les critères à la lumière de l'histoire. In D. Lhuilier, (Ed.), Qualité du travail, qualité au travail (pp. 17-25). Toulouse : Octarès.

Lhuilier, D. (2014). Introduction. In D. Lhuilier, (Ed.), Qualité du travail, qualité au travail. (pp. 3-14), Toulouse : Octarès.

Livet, P. (2002). Émotions et rationalité morale. Paris : PUF.

Lorino, P. (2013). L'activité collective, processus organisant : un processus discursif fondé sur le langage pragmatiste des habitudes. Activités, 10(1), 221-242. En ligne. URL : https:// activites.revues.org/656

Moscovici, S. (1991). Psychologie des minorités actives. Paris : PUF.

Moscovici, S. (1994). Psychologie sociale des relations à autrui. Paris : Nathan.

Nascimento, A., \& Falzon, P. (2009). Produire la santé, produire la sécurité. Récupérations et compromis dans le risque des manipulatrices en radiothérapie. Activités, 6(2), 3-23. En ligne. URL : https://activites.revues.org/2225

Perez Toralla, M.S. (2013). Pour une prescription capacitante : ergonomie et débats des règles du travail. Le cas d'une entreprise déployant la Lean production. Thèse de doctorat en ergonomie, Paris : CNAM.

Perrot, E. (2017). L'activité transférentielle dans l'intervention. Le cas des agents de gare à la SNCF. Thèse pour le doctorat de psychologie. Paris : CNAM.

Petit, J., Dugué, B., \& Daniellou, F. (2011). L'intervention ergonomique sur les risques psychosociaux dans les organisations : Enjeux théoriques et méthodologiques. Le travail humain, 74(4), 391-409.

Poussin, N. (2014). Développement des sentiments au travail : dialogues sur l'efficacité et l'utilité chez des médecins du travail. Thèse pour le doctorat de psychologie. Paris : CNAM.

Poussin, N. (2015). Développement du sentiment d'efficacité : un développement du rapport entre affectivité et pensée. Activités, 12(1), 89-103. En ligne. URL : https://activites.revues.org/1028

Prot, B. (2006). La fonction du clinicien dans les méthodes de clinique de l'activité. Nouvelle revue de psychosociologie, 1(1). 31-44.

Quillerou-Grivot, E. (2011). Fonction psychologique et sociale du collectif pour la santé au travail : le cas de l'activité d'opérateurs de montage automobile. Thèse pour le doctorat en psychologie. Paris : CNAM.

Quillerou-Grivot, E., \& Clot, Y. (2014). La qualité du travail : un problème avant d'être une solution. In D. Lhuilier, (Ed.), Qualité du travail, qualité au travail. (pp. 141-159), Toulouse : Octarès. Rabardel, P. 1995. Les hommes et les technologies. Paris : Armand Colin.

Sarnin, P., Bobillier-Chaumon, M.E., Cuvillier, B., \& Grosjean, M. (2012). Intervenir sur les souffrances au travail : acteurs et enjeux dans la durée. Bulletin de psychologie, 519(3). 251-261. 
Simonet, P., Caroly, S., \& Clot, Y. (2011). Méthodes d'observation de l'activité de travail et prévention durable des TMS. Activités, 8(1). En ligne. URL : https://activites.revues.org/2481

Terssac, G. (de) (2011). Théorie du travail d'organisation. In B. Maggi, (Ed.), Interpréter l'agir : un défi théorique (pp. 97-121). Paris : PUF.

Tomás, J.L., \& Bonnefond, J.Y. (2014). De « l'épuisement professionnel » à la qualité du travail : le cas de la «belle table d'instrumentation ». In D. Lhuilier, (Ed.), Qualité du travail, qualité au travail, (pp. 129-140). Toulouse : Octarès.

Tosquelles, F. (2009). Le travail thérapeutique en psychiatrie. Toulouse : Erès.

Vygotski, L. (1978). Mind in society : the development of higher psychological processes. Cambridge : Harvard University Press.

Vygotski, L. (1994). Défectologie et déficience mentale. Textes publiés par K. Barisnikov et G. Petitpierrre. Neuchatel : Delachaux et Niestlé.

Vygotski, L. (1997). Pensée et Langage. (F. Sève, trad.). Paris : La dispute.

Vygotski, L. (2003). Conscience, inconscient, émotions. (F. Sève \& G. Fernandez, trad.). Paris : La

Dispute.

Vygotski, L. (2014). Histoire du développement des fonctions psychiques supérieure (F. Sève, trad.).

Paris : La Dispute.

Wolmark, C. (2016). Le travail absent du droit du travail ? Travailler, 36, 155-173.

\section{NOTES}

1. Pour une discussion sur cette perspective, voir Clot, Y., Bouffartigue, P., Durand, J.P., Fortino, S \& Mias, A. (2016)

2. Nous entendons par là, à la fois que l'action recherche délibérément le développement du professionnalisme à tous les niveaux de l'organisation et qu'elle le fait en développant la délibération collective.

3. Opérateur expérimenté, extérieur à la chaine et qui intervient, à la demande, sur les problèmes d'approvisionnement, sur les retouches et sur les outils.

4. On cherche un autre centre de gravité au rapport social habituel : le réel du travail

5. Nature des opérations à réaliser dans le temps de cycle

6. Cotation de gravité des défauts de V1+ à V3. V2 = défaut d'aspect

7. C'est ainsi que Vygotski définit la «Zone de Développement Potentielle » $(1997,2014)$

8. Les sujets sont dits sensibles lorsqu'ils sont évalués comme importants au regard de la performance ou de la sécurité et/ou que leur traitement s'éternise.

9. Des expérimentations sociales du même genre sont en cours dans le cadre de conventions du CRTD-CNAM (Municipalité de Lille, CNFPT, AFPA, EPHAD de Grugny). Ces développements de l'intervention méritent sans doute d'être regardés sous l'angle des méthodes de clinique de l'activité en psychologie du travail. 


\section{RÉSUMÉS}

Cet article étudie un développement organisationnel au service de la santé et de la performance en psychologie du travail. À partir d'une intervention à l'usine Renault de Flins, nous étudions comment le cadre clinique a donné lieu à des développements allant jusqu'à la conception et l'institution d'un dispositif organisationnel de dialogue sur la qualité du travail (DQT) à l'échelle de l'usine, instrumentant le dialogue et l'action sur les problèmes du travail. Cet exemple documente l'importance d'une méthodologie qui fasse du développement personnel, collectif et organisationnel à la fois l'objet et la méthode de l'intervention. En effet, il montre que, dans une perspective de santé et de performance, pour développer l'organisation, il faut pouvoir expérimenter sa transformation par les professionnels concernés au cours de l'intervention. En outre, ce dispositif qui a donné lieu à une nouvelle fonction d'opérateur référent-métier, met en lumière l'importance d'enrichir la division technique et hiérarchique existante pour faire des conflits de critères sur la qualité du travail la source de l'efficacité et de la santé dans l'organisation.

This article examines an organizational development for health and performance in work psychology. Based on an intervention at the Renault Flins factory, we study how the clinical framework has led to the design and generalization of an organizational device for dialogue on work quality which provides sustainable dialogue and action on work issues in the factory. This case shows the importance of a methodology that makes personal, collective and organizational development both the object and the method of intervention. Indeed, from a health and performance perspective it shows that in order to develop the organization, it is necessary for the professionals concerned to experiment on its transformation during the intervention. Moreover, this system, which has given rise to a new function as a referent operator, underlines the importance of improving the existing technical and hierarchical division to make conflicts of criteria on the quality of work, the source of efficiency and health in the organization.

\section{INDEX}

Keywords : quality of work, criteria's conflict, intervention, development, organization, health and performance

Mots-clés : qualité du travail, conflit de critères, intervention, développement, organisation, performance et santé

\section{AUTEUR}

\section{JEAN-YVES BONNEFOND}

CNAM, Équipe Psychologie du travail et clinique de l'activité, CRTD, 41, rue Gay-Lussac, 75005

Paris. jeanyves.bonnefond@cnam.fr 